\title{
THREE DIMENSIONAL MHD CASSON FLUID FLOW OVER A STRETCHING SURFACE WITH VARIABLE THERMAL CONDUCTIVITY
}

\author{
B. Ganga ${ }^{1}$, S. Charles ${ }^{2}$, A.K. Abdul Hakeem ${ }^{3}$, S. Nadeem ${ }^{4,5}$ \\ ${ }^{1}$ Department of Mathematics, Providence College for Women, Coonoor-643104, India \\ ${ }^{2}$ Department of Mathematics, PSG College of Arts and Science, Coimbatore-641014, India \\ ${ }^{3}$ Department of Mathematics, SRMV College of Arts and Science, Coimbatore - 641020, India \\ ${ }^{4}$ Mathematics and its Applications in Life Sciences Research Group, Ton Duc Thang University, \\ Ho Chi Minh City, Vietnam \\ ${ }^{5}$ Faculty of Mathematics and Statistics, Ton Duc Thang University, Ho Chi Minh City, Vietnam \\ gangabhose@gmail.com
}

Received: 15 August 2020; Accepted: 1 March 2021

\begin{abstract}
The three-dimensional magnetohydrodynamic (MHD) boundary layer flow of a Casson fluid over a stretching surface set into a porous medium with variable thermal conductivity and heat generation/absorption has been researched. Conservation laws of mass, momentum and energy are changed into ordinary differential equations, which are numerically dealt with by applying the fourth order Runge-Kutta integration scheme in relationship with shooting procedure. The dimensionless velocity, temperature, skin friction coefficient and the local Nusselt number inside the boundary layer are processed and examined through tables and illustrations for various physical parameters. The numerical outcomes obtained for the specific case are sensible in great concurrence with the existing results. Results indicate that momentum boundary layer reduces for the Hartman number and Casson fluid parameter. Temperature is found as an enlightened function for the heat generation and thermal conductivity parameter.
\end{abstract}

MSC 2010: 76D05, 76W05, 80A20

Keywords: three-dimensional flow, Casson fluid, stretching surface, heat generation/ labsorption

\section{Introduction}

It is a verifiable truth that the Casson fluid model is a decent contender to investigate their properties of non-Newtonian fluids, a large number of research papers are identified in literature survey, that corresponds with improving the thermal conductivity of the Casson fluid (Haldar et al. [1], Sivaraj et al. [2], Hamid et al. [3], Gireesha et al. [4], Satya Narayana et al. [5], Eid and Mahny [6], Indumathi et al. [7], Tarakaramu et al. [8], Abdul Hakeem et al. [9], and Eid and Mahny [10]). Many 
researchers find variable thermal conductivity under different flow geometries. In the literature, variable thermal conductivity towards various geometries is widely available. For instance, Sarojamma et al. [11], Kezzar et al. [12], Nawaz et al. [13], Salahuddin et al. [14], Pinarbasia et al. [15], Sekhar et al. [16] and Vaidya et al. [17] are a few of the recent research.

The 3-D flow of non-Newtonian fluids via the stretching surface also has the majority of applications in civil engineering, solar energy and peristalsis blood flow through the valves. In a suspension of graphene nanoparticles, Durgaprasad et al. [18] studied the combined effects of Brownian motion and thermophoresis parameters on Casson's three-dimensional nanofluid flow across porous layers slendering sheets. Raju et al. [19] investigated the flow of heat and mass transfer in Williamson-Casson 3-D fluids over a stretching surface with non-uniform heat sink/source. Prashu [20] found numerical treatment of unstable, three-dimensional hydromagnetic flow with Hall and radiation effects of a Casson fluid. Muhammad et al. [21] addressed Casson nanofluid's three-dimensional flow over a stretched surface with chemical reactions, velocity fall, thermal radiation and Brownian motion. Similarly, with the effect of heat generation/consumption and thermal radiation, Saeed et al. [22] addressed the three-dimensional Casson nanofluid thin film flow over an inclined rotational disk. Prasad et al. [23] carried out a study on the three-dimensional slip flow of a chemically reacting Casson fluid flowing over a porous slender sheet with a non-uniform heat source or sink.

A close observation toward this path reveals that so far no one has considered building a mathematical model for MHD three-dimensional flow of Casson fluid with variable thermal conductivity over a stretching surface. Remembering this an investigation has been done with the said problem. Results are plotted and displayed. The important observations of investigation are recorded in the conclusions.

\section{Mathematical formulation}

Consider the existence of variable thermal conductivity to a steady, three-dimensional boundary layer flow of an incompressible Casson fluid. The fluid conducts electrically under the influence of a constant applied magnetic field. Constants are considered for physical properties of the substance. Effects of an induced magnetic field and viscous dissipation are neglected. We denote $T$ the temperature and $T_{\infty}$ the ambient temperature, respectively. The physical configuration is given in Figure 1. The resulting boundary layer equations are (Eid and Mahny [11], Sarojamma et al. [12] and Durgaprasad et al. [18])

$$
\begin{gathered}
\frac{\partial u}{\partial x}+\frac{\partial v}{\partial y}+\frac{\partial w}{\partial z}=0 \\
u \frac{\partial u}{\partial x}+v \frac{\partial u}{\partial y}+w \frac{\partial u}{\partial z}=v\left(1+\frac{1}{\beta}\right) \frac{\partial^{2} u}{\partial z^{2}}-\frac{\sigma^{*} B_{0}^{2} u}{\rho}-\frac{v}{K} u
\end{gathered}
$$




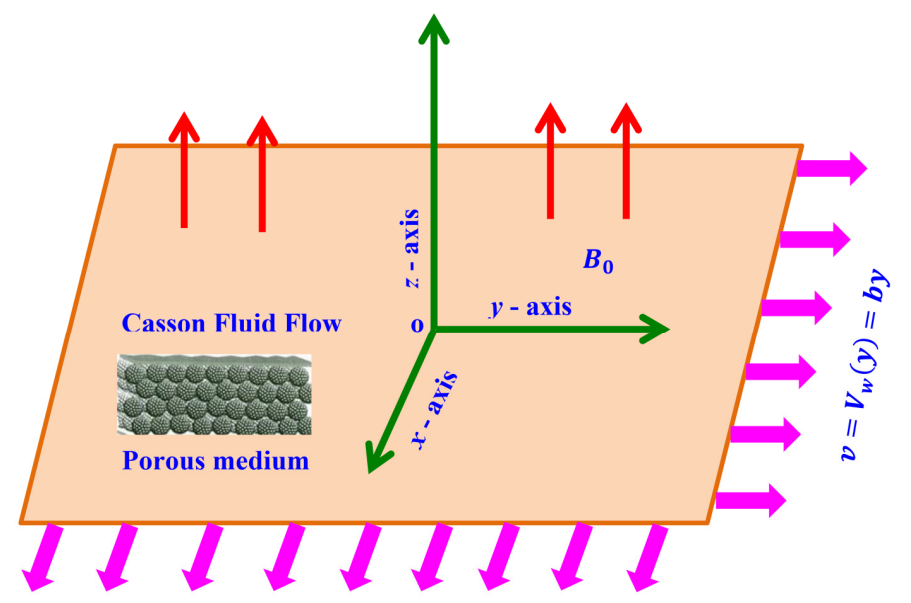

$u=U_{w}(x)=a x$

Fig. 1. Physical configuration

$$
\begin{gathered}
u \frac{\partial v}{\partial x}+v \frac{\partial v}{\partial y}+w \frac{\partial v}{\partial z}=v\left(1+\frac{1}{\beta}\right) \frac{\partial^{2} v}{\partial z^{2}}-\frac{\sigma^{*} B_{0}^{2} v}{\rho}-\frac{v}{K} v \\
u \frac{\partial T}{\partial x}+v \frac{\partial T}{\partial y}+w \frac{\partial T}{\partial z}=\frac{1}{\rho C_{p}} \frac{\partial}{\partial z}\left(k^{*} \frac{\partial T}{\partial z}\right)+\frac{Q}{\rho C_{p}}\left(T-T_{\infty}\right)
\end{gathered}
$$

Where $u, v$ and $w$ the $x, y$ and $z$ components of velocity, $\beta=\mu_{B} \sqrt{\frac{2 \pi_{p}}{p_{y}}}$ is the Casson fluid parameter, $\sigma^{*}-$ the electrical conductivity, $K$ - the permeability parameter, $C_{p}$ - the specific heat, $\sigma$ is the thermal diffusivity of the fluid, $v=\frac{\mu_{B}}{\rho}-$ the kinematic viscosity, $\mu_{B}$ - the plastic dynamic viscosity of Casson fluid, $\rho$ - the density of fluid and $Q$ is the volumetric heat generation/absorption coefficient.

The corresponding boundary conditions are:

$$
\begin{gathered}
u=a x, v=b y, w=0, k \frac{\partial T}{\partial z}=h_{w}\left(T_{w}-T\right), \text { at } z=0 \\
u \rightarrow 0, v \rightarrow 0, \mathrm{~T} \rightarrow \mathrm{T}_{\infty} \text { as } z \rightarrow \infty
\end{gathered}
$$

where the fluid temperature of the wall is $T_{w}$. Employing the transformations (Shehzad et al. [24])

$$
\begin{gathered}
u=a x f^{\prime}(\eta), v=a y g^{\prime}(\eta), w=-\sqrt{a} v(f(\eta)+g(\eta)), \\
\theta(\eta)=\frac{T-T_{\infty}}{T_{w}-T_{\infty}}, \eta=z \sqrt{\frac{a}{v}}
\end{gathered}
$$


One obtains

$$
\begin{gathered}
\left(1+\frac{1}{\beta}\right) f^{\prime \prime \prime}+(f+g) f^{\prime \prime}-f^{\prime 2}-\left(M^{2}+\frac{1}{\lambda}\right) f^{\prime}=0 \\
\left(1+\frac{1}{\beta}\right) g^{\prime \prime \prime}+(f+g) g^{\prime \prime}-g^{\prime 2}-\left(M^{2}+\frac{1}{\lambda}\right) g^{\prime}=0 \\
(1+\in \theta) \theta^{\prime \prime}+\in \theta^{\prime 2}+\operatorname{Pr}(f+g) \theta^{\prime}+\operatorname{Pr} B \theta=0 \\
f=0, g=0, f^{\prime}=1, g^{\prime}=\alpha, \theta=1 \text { at } \eta=0 \\
f^{\prime} \rightarrow 0, g^{\prime} \rightarrow 0, \theta \rightarrow 0 \text { as } \eta \rightarrow \infty
\end{gathered}
$$

where Eq. (1) is satisfied automatically, $M^{2}=\frac{\sigma^{*} B_{0}^{2}}{a \rho}$ is the Hartman number, $\lambda=\frac{K a}{v}-$ the porosity parameter, $\alpha=\frac{b}{a}$ is a ratio parameter, $\operatorname{Pr}=\frac{v}{\sigma}-$ the Prandtl number and $B=\frac{Q}{\rho a C_{p}}$ is the heat generation/absorption coefficient and prime depicts differentiation with respect to $\eta$.

If $C_{f x}$ and $C_{f y}$ are the skin-friction coefficients and $N_{u x}$ is the local Nusselt number, then we have

$$
C_{f x}=\frac{\tau_{w} x}{\rho u_{w}^{2}}, C_{f y}=\frac{\tau_{w} y}{\rho u_{w}^{2}}, N u_{x}=\frac{x q_{w}}{k\left(T_{w}-T_{\infty}\right)}
$$

where $\tau_{w}$ and $q_{w}$ are the wall shear stress and the wall heat flux, respectively. The above equation in its dimensionless form can be written as

$$
\begin{aligned}
\operatorname{Re}_{x}^{-\frac{1}{2}} C_{f x} & =\left(1+\frac{1}{\beta} f^{\prime \prime}(0)\right), \operatorname{Re}_{x}^{-\frac{1}{2}} C_{f y}=\left(1+\frac{1}{\beta} g^{\prime \prime}(0)\right), \\
\operatorname{Re}_{x}^{-\frac{1}{2}} N u_{x} & =-\left(\theta^{\prime}(0)\right)
\end{aligned}
$$

where the definition of the Reynolds number is $\operatorname{Re}_{x}=\frac{u_{w x}}{v}$.

\section{Numerical solution}

The system of coupled nonlinear coupled differential equations (6) and (7), along with the boundary conditions (8) and (9), is solved numerically using the fourth-order 
Runge-Kutta method with a shooting technique (Satya Narayana et al. [6], Eid et al. [25]). The system of equations (6)-(9) is reduced with this approach to a system of first order initial value issues. This boundary value problem is solved using the above approaches as initial value issues. The above-mentioned Runge-Kutta method with a shooting technique is used to get the unknowns $f^{\prime \prime}(0), g^{\prime \prime}(0)$ and $\theta^{\prime}(0)$ up to the boundary conditions $f^{\prime} \rightarrow 0, g^{\prime} \rightarrow 0, \theta \rightarrow 0$ as $\eta \rightarrow \infty$ satisfied. The phase size $\Delta \eta=0.001$ is used to achieve the numerical solution with gmax, and accuracy to the fifth decimal place is used as the criterion of convergence.

\section{Results and discussion}

In the present section, we have discussed the velocity profiles, temperature profile, skin friction coefficient and Nusselt number for various physical parameters such as Casson parameter $\beta$, Hartmann number $M$, porosity parameter $\lambda$, thermal conductivity parameter $\varepsilon$ and ratio parameter $\alpha$.

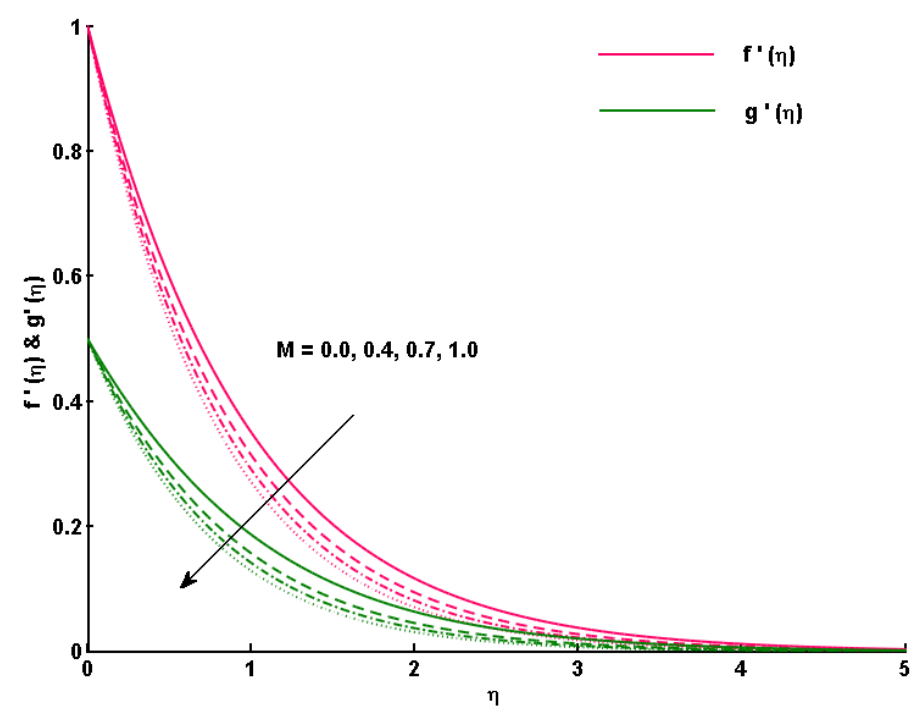

Fig. 2. Influence of $M$ on $f^{\prime}(\eta)$ and $g^{\prime}(\eta)$ when $\beta=1.5, \lambda=2.0$ and $\alpha=0.5$

Figures 2 and 3 are displayed to investigate the impacts of Hartman number $M$ and Casson parameter $\beta$ on the velocities and $g^{\prime}(\eta)$. Here, it is observed that an expansion in the Hartman number decreases the fluid velocities and their related momentum boundary layer thickness. Physically, $M$ creates a stronger Lorentz force on the fluid in the specified domain, which lessen the movement of the fluid. Subsequently, in Figure $3, f^{\prime}(\eta)$ and $g^{\prime}(\eta)$ and the boundary layer thickness diminishes for higher estimations of $\beta$. In addition, it is noted that the Casson parameter has no effect when the fluid is moving outside the solid boundaries. 


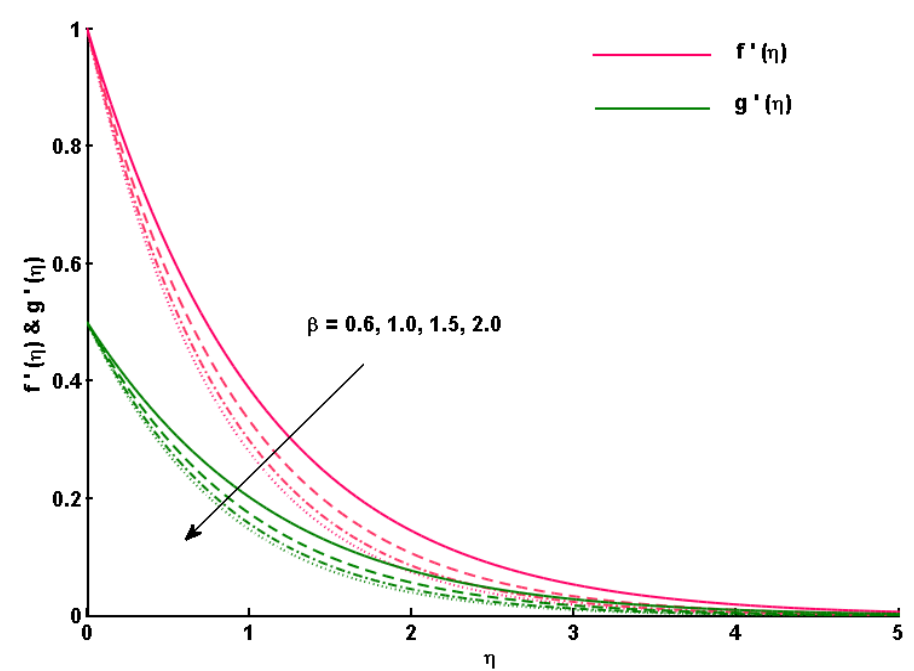

Fig. 3. Influence of $\beta$ on $f^{\prime}(\eta)$ and $g^{\prime}(\eta)$ when $M=0.6, \lambda=2.0$ and $\alpha=0.5$

Figure 4 delineates how the ratio parameter $\alpha$ and porosity parameter $\lambda$ influence the temperature profile. With the amplified estimation of the temperature, $\theta^{\prime}(\eta)$ decreases. In this progression, the impacts of heat generation/absorption parameter $B$ and thermal conductivity parameter $\varepsilon$ on the temperature profile is shown in Figure 5 respectively. This depicts that, and $\varepsilon$ has similar effects on the temperature. Growing porosity often widens the thermal boundary layer.

At the point when heat generation parameter $B$ becomes greater than before, more heat is delivered in the fluid that results in sa higher temperature and higher thermal boundary layer thickness. Likewise, it is seen that the impact of $\varepsilon$ improves the temperature profile, essentially.

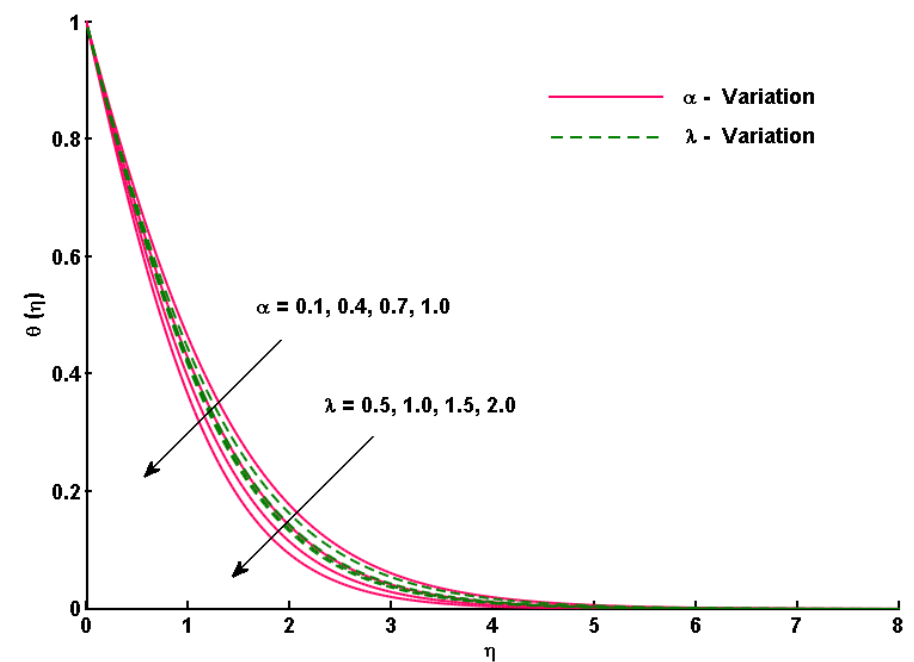

Fig. 4. Influence of $\alpha$ and $\lambda$ on $\theta(\eta)$ when $M=0.6, \beta=1.5, \operatorname{Pr}=0.9, B=0.4$ and $\varepsilon=0.7$ 


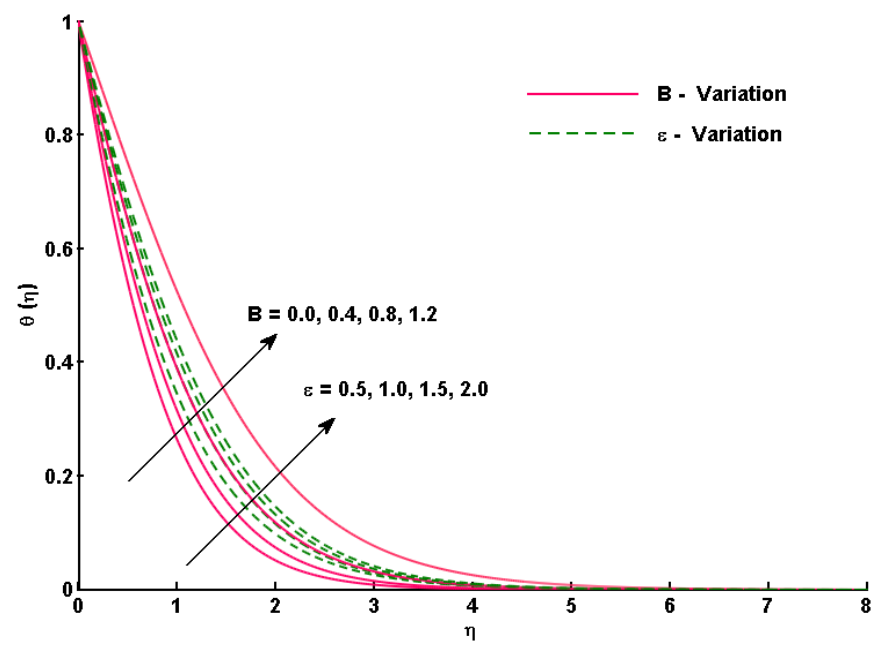

Fig. 5. Influence of $B$ and $\varepsilon$ on $\theta(\eta)$ when $M=0.6, \lambda=2.0, \beta=1.5, \operatorname{Pr}=0.9, \alpha=0$

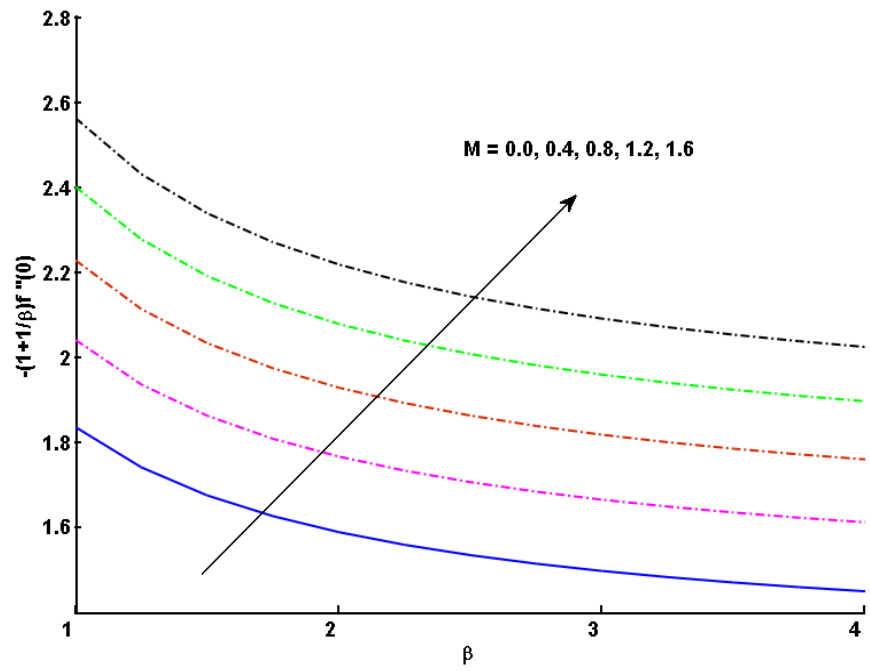

Fig. 6. Influence of $M$ and $\beta$ on $\left(1+\frac{1}{\beta}\right) f^{\prime \prime}(0)$ when $\operatorname{Pr}=0.9, B=0.4, \lambda=2.0, \varepsilon=0.7$ and $\alpha=0.5$

Figures 6 and 7 are drawn up to see the results of the Hartman number on the skinfriction coefficients $\left(1+\frac{1}{\beta}\right) f^{\prime \prime}(0),\left(1+\frac{1}{\beta}\right) g^{\prime \prime}(0)$. A comparison of Figures 6 and 7 shows that the skin-friction coefficient $\left(1+\frac{1}{\beta}\right) f^{\prime \prime}(0)$ at the wall are greater than the skin-friction coefficient $\left(1+\frac{1}{\beta}\right) g^{\prime \prime}(0)$. 


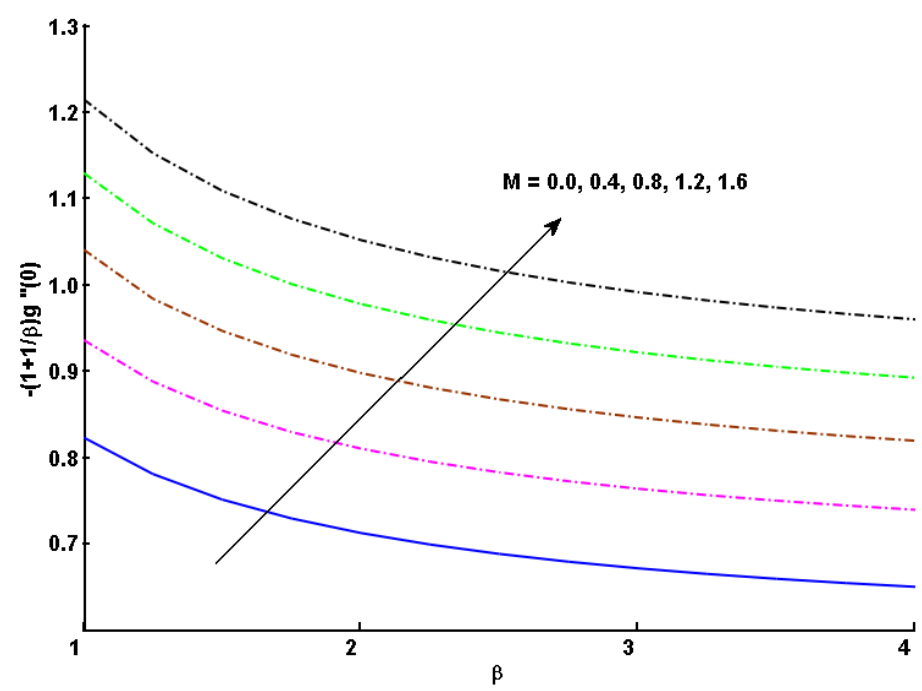

Fig. 7. Influence of $M$ and $\beta$ on $\left(1+\frac{1}{\beta}\right) g^{\prime \prime}(0)$ when $\operatorname{Pr}=0.9, B=0.4, \lambda=2.0, \varepsilon=0.7$ and $\alpha=0.5$

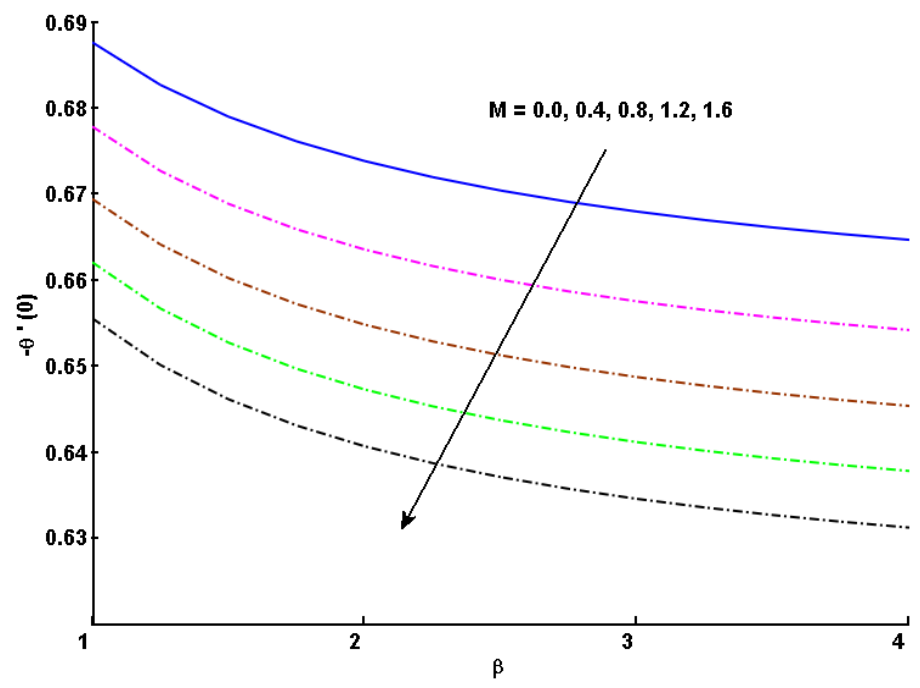

Fig. 8. Influence of $M$ and $\beta$ on $\theta^{\prime}(0)$ when $\operatorname{Pr}=0.9, B=0.4, \lambda=2.0, \varepsilon=0.7$ and $\alpha=0.5$

The local Nusselt number is introduced in Figures 8-10 under the impacts of distinct physical parameters. Figure 8 displays the combined effects of Hartman number along with the Casson parameter. One can observe that the increment of Hartman number and the Casson parameter result to decrease the local Nusselt number. An expansion in heat generation/absorption parameter prompts an increment in the local Nusselt number which we see from Figure 9. 


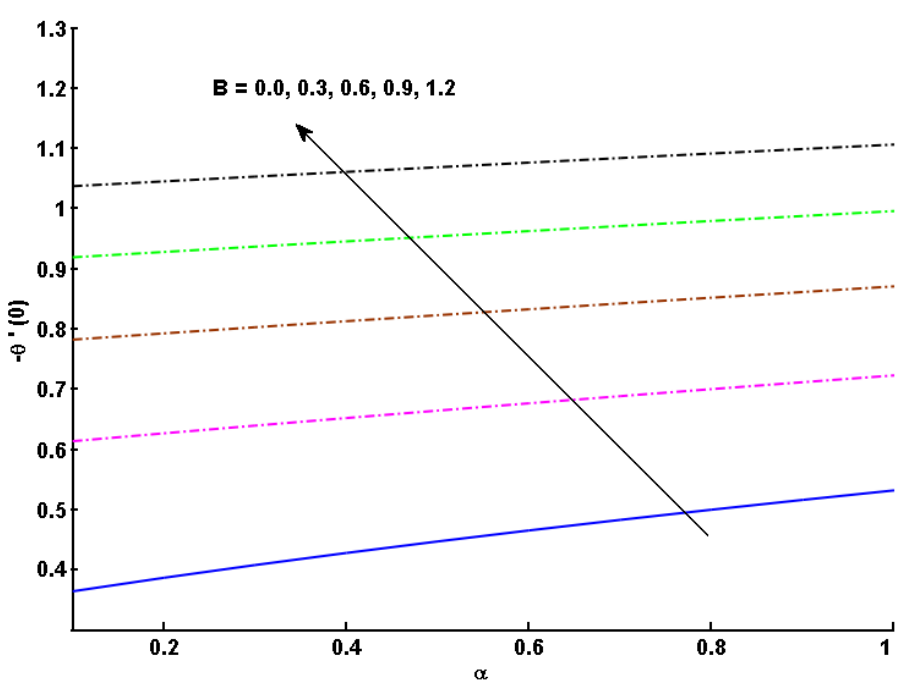

Fig. 9. Influence of $B$ and $\alpha$ on $\theta^{\prime}(0)$ when $\operatorname{Pr}=0.9, M=0.6, \lambda=2.0, \varepsilon=0.7$ and $\beta=1.5$

Figure 10 demonstrates the varieties in the local Nusselt number $-\theta^{\prime}(0)$ for various estimations of $\lambda$ vs $\alpha$. For $\lambda$ with $\alpha$, the local Nusselt number acts as an increasing function.

Table 1 shows that the awesome correlation with the earlier work has been identified by Shezhad [24] for $f^{\prime \prime}(0), g^{\prime \prime}(0)$ and $\theta^{\prime}(0)$ in the absence of both the Casson fluid parameter $\beta$, porosity parameter $\lambda$ and thermal conductivity parameter $\varepsilon$. It is found to be in good agreement, which is shown in Table 1 .

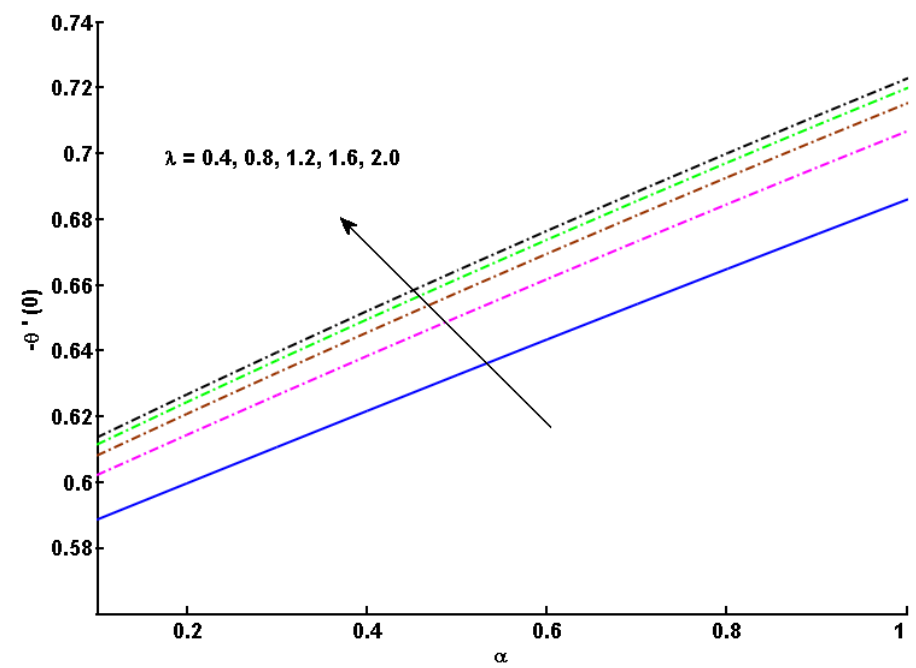

Fig. 10. Influence of $\lambda$ and $\alpha$ on $\theta^{\prime}(0)$ when $\operatorname{Pr}=0.9, M=0.6, B=0.3, \varepsilon=0.7$ and $\beta=1.5$ 
Table 1. Comparison of results for the local skin friction coefficient $f^{\prime \prime}(0)$ and $g^{\prime \prime}(0)$ for different value of $\alpha$ when $\beta \rightarrow \infty, \lambda \rightarrow \infty$, and $M=0$

\begin{tabular}{|c|c|c|c|c|}
\hline \multirow{2}{*}{$\mathrm{A}$} & \multicolumn{2}{|c|}{ Shehzed [24] } & \multicolumn{2}{c|}{ Present results } \\
\cline { 2 - 5 } & $-f^{\prime \prime}(0)$ & $-g^{\prime \prime}(0)$ & $-f^{\prime \prime}(0)$ & $-g^{\prime \prime}(0)$ \\
\hline 0.0 & 1 & 0 & 1 & 0 \\
\hline 0.25 & 1.04881 & 0.19457 & 1.048810976 & 0.194563936 \\
\hline 0.50 & 1.09309 & 0.46522 & 1.093093692 & 0.465208195 \\
\hline 0.75 & 1.13450 & 0.79462 & 1.134484180 & 0.794620731 \\
\hline 1.0 & 1.17372 & 1.17372 & 1.173721027 & 1.173721027 \\
\hline
\end{tabular}

Table 2 is computed to analyze the numerical values of $f^{\prime \prime}(0), g^{\prime \prime}(0)$ and $\theta^{\prime}(0)$ for the involved parameters. Here we have seen that the skin friction coefficient increases for Hartman number $M$ and ratio parameter $\alpha$ and decreasing with other parameters while the Nusselt number increases for ratio parameter $\alpha$, heat generation parameter $B$ and porosity parameter $\lambda$, and reduce for all other parameters.

Table 2. Values of skin friction and Nusselt number for various values of governing parameters

\begin{tabular}{|c|c|c|c|c|}
\hline \multirow{3}{*}{ Parameter } & Values & $-\left(1+\frac{1}{\beta}\right) f^{\prime \prime}(0)$ & $-\left(1+\frac{1}{\beta}\right) g^{\prime \prime}(0)$ & $-\theta^{\prime}(0)$ \\
\hline \multirow{3}{*}{$M$} & 0.4 & 1.86292359 & 0.85396893 & 0.66917935 \\
\cline { 2 - 5 } & 0.8 & 2.03276180 & 0.94577244 & 0.66071787 \\
\hline \multirow{2}{*}{$\alpha$} & 0.4 & 1.93429467 & 0.70161804 & 0.65256802 \\
\cline { 2 - 5 } & 0.7 & 1.97977661 & 1.32582151 & 0.68860669 \\
\hline \multirow{2}{*}{$\beta$} & 0.5 & 2.61797124 & 1.21022345 & 0.69238328 \\
\hline \multirow{2}{*}{$\varepsilon$} & 1.0 & 2.13594816 & 0.98714161 & 0.67371713 \\
\cline { 2 - 5 } & 0.5 & 1.94962042 & 0.90099289 & 0.70616291 \\
\hline \multirow{2}{*}{$B$} & 1.0 & 1.94962042 & 0.90099289 & 0.61843015 \\
\cline { 2 - 5 } & 0.3 & 1.94962042 & 0.90099289 & 0.66479190 \\
\hline \multirow{2}{*}{$\lambda$} & 0.6 & 1.94962042 & 0.90099289 & 0.82249665 \\
\cline { 2 - 5 } & 1.6 & 2.00197472 & 0.92922411 & 0.66221130 \\
\hline
\end{tabular}

Note: While studying the individual parameters the following values are assumed $M=0.6, \alpha=0.5$, $B=0.3, \varepsilon=0.7, \beta=1.5, \lambda=2.0$

\section{Conclusion}

The main results of present study can be summarized as follows:

- Hartman number $M$ and Casson fluid parameter reduce the velocities $f^{\prime}(\eta)$ and $g^{\prime}(\eta)$. 
- Heat generation parameter $B$ and thermal conductivity parameter $\varepsilon$ increase the temperature profile. On the other hand, temperature profile is reduced by the ratio parameter $\alpha$ and porosity parameter $\lambda$.

- The magnitude of the skin friction coefficients in both directions increases with an increase in both the Hartmann number $M$ and ratio parameter $\alpha$, respectively and shows an opposite effect for Casson parameter $\beta$ and porosity parameter $\lambda$.

- The rate of heat transfer was found to be higher for ratio parameter $\alpha$, heat generation parameter $B$ and porosity parameter $\lambda$.

\section{References}

[1] Haldar, S., Mukhopadhyay, S., \& Layek, G.C. (2019). Flow and heat transfer of Casson fluid over an exponentially shrinking permeable sheet in presence of exponentially moving free stream with convective boundary condition. Mechanics of Advanced Materials and Structures, 26(17), 1498-1504.

[2] Sivaraj, R., Jasmine Benazir, A., Srinivas, S., \& Chamkha, Ali J. (2019). Investigation of crossdiffusion effects on Casson fluid flow in existence of variable fluid properties. The European Physical Journal Special Topics, 228(1), 35-53.

[3] Hamid, M., Usman, M., Khan, Z.H., Ahmad, R., \& Wang, W. (2019). Dual solutions and stability analysis of flow and heat transfer of Casson fluid over a stretching sheet. Physics Letters A, 383(20), 2400-2408.

[4] Gireesha, B.J., Archana, M., Mahanthesh, B., \& Prasannakumara, B.C. (2019). Exploration of activation energy and binary chemical reaction effects on nano Casson fluid flow with thermal and exponential space-based heat source. Multidiscipline Modeling in Materials and Structures, 15(1), 227-245.

[5] Satya Narayana, P.V., \& Harish Babu, D. (2015). Numerical study of MHD heat and mass transfer of a Jeffrey fluid over a stretching sheet with chemical reaction and thermal radiation. Journal of the Taiwan Institute of Chemical Engineers, 59, 18-25.

[6] Eid, M.R., Mahny, K.L. (2017). Flow and heat transfer in a porous medium saturated with a Sisko nanofluid over a nonlinearly stretching sheet with heat generation/absorption. Heat Transfer-Asian Research, 47(1), 54-71.

[7] Indumathi, N., Abdul Hakeem, A.K., Ganga, B., Jayaprakash, R. (2021). Marangoni convection of titanium dioxide/ethylene glycol dusty nanoliquid MHD flow past a flat plate. Advances in Fluid Dynamics. Lecture Notes in Mechanical Engineering, DOI: 10.1007/978-981-15-4308-1_19.

[8] Tarakaramu, N., \& Satya Narayana, P.V. (2021). Influence of heat generation/absorption on 3D magnetohydrodynamic Casson fluid flow over a porous stretching surface. Advances in Fluid Dynamics. Lecture Notes in Mechanical Engineering, DOI: 10.1007/978-981-15-4308-1_30.

[9] Abdul Hakeem, A.K., Renuka, P., Vishnu Ganesh, N., Kalaivanan, R., \& Ganga, B. (2016). Influence of inclined Lorentz forces on boundary layer flow of Casson fluid over an impermeable stretching sheet with heat transfer. Journal of Magnetism and Magnetic Materials, 401, 354-361.

[10] Eid, M.R., \& Mahny, K.L. (2017). Unsteady MHD heat and mass transfer of a non-Newtonian nanofluid flow of a two-phase model over a permeable stretching wall with heat generation/ absorption. Advanced Powder Technology, 28(11), 3063-3073.

[11] Sarojamma, G., Sreelakshmi, K., Krishna Jyothi, P., \& Satya Narayana, P.V. (2020). Influence of homogeneous and heterogeneous chemical reactions and variable thermal conductivity on the MHD Maxwell fluid flow due to a surface of variable thickness. Defect and Diffusion Forum, 401, 148-163. 
[12] Kezzar, M., Tabet, I., \& Eid, M.R. (2020). A new analytical solution of longitudinal fin with variable heat generation and thermal conductivity using DRA. European Physical Journal Plus, 135, 120, DOI: 10.1140/epjp/s13360-020-00206-0.

[13] Nawaz, M., Rahila Naz, \& Awais, M. (2018). Magnetohydrodynamic axisymmetric flow of Casson fluid with variable thermal conductivity and free stream. Alexandria Engineering Journal, 57(3), 2043-2050.

[14] Salahuddin, T., Malik, M.Y., Arif Hussain, Bilal S., \& Awais M. (2016). Combined effects of variable thermal conductivity and MHD flow on pseudoplastic fluid over a stretching cylinder by using Keller box method, Information Sciences Letters, 5(1), 11-19.

[15] Pinarbasia, A., Ozalp C., \& Duman, S. (2005). Influence of variable thermal conductivity and viscosity for nonisothermal fluid flow, Physics of Fluids, 17, 038109.

[16] Sekhar, K.R., Thajoddin, S., Reddy, G.V., \& Bhaskarudu, P. (2017). Effects of heat source/sink on MHD flow of Casson fluid and heat transfer over an unsteady stretching sheet. International Journal of Innovative Research in Science, Engineering and Technology, 6(5), 7835-7844.

[17] Vaidya, H., Rajashekhar, C., Manjunatha, G., Prasad, K.V., Makinde O.D., \& Sreenadh, S. (2019). Peristaltic motion of non-Newtonian fluid with variable liquid properties in a convectively heated non-uniform tube: Rabinowitsch fluid model. Journal of Enhanced Heat Transfer, 26(3), 277-294.

[18] Durgaprasad, P., Varma, S.V.K., \& Hoque, M.M. (2019). Combined effects of Brownian motion and thermophoresis parameters on three-dimensional (3D) Casson nanofluid flow across the porous layers slendering sheet in a suspension of graphene nanoparticles. Neural Computing \& Applications Journal, 31, 6275-6286.

[19] Raju, C.S.K., Sandeep, N., Ali, M.E., \& Nuhait, A.O. (2019). Heat and mass transfer in 3D Williamson-Casson fluids flow over a stretching surface with non-uniform heat source/sink. Thermal Science, 23(1), 281-293.

[20] Prashu, R.N. (2018). A numerical treatment of unsteady three-dimensional hydromagnetic flow of a Casson fluid with Hall and radiation effects. Results in Physics, 11, 966-974.

[21] Muhammad, U., Zulqurnain, S., Ali, I., Hafiz, W.A., Muhammad, S., \& Muhammad Asif Zahoor, R. (2019). Three-dimensional flow of Casson nanofluid over a stretched sheet with chemical reactions, velocity slip, thermal radiation and Brownian motion. Thermal Science, 339.

[22] Saeed, A., Shah, Z., Islam, S., Jawad, M., Ullah, A., Gul, T., \& Kumam, P. (2019). Threedimensional Casson nanofluid thin film flow over an inclined rotating disk with the impact of heat generation/consumption and thermal radiation. Coatings, 9, 248.

[23] Prasad, P.D., Saleem, S., \& Varma, S.V.K. (2019). Three dimensional slip flow of a chemically reacting Casson fluid flowing over a porous slender sheet with a non-uniform heat source or sink. Journal of the Korean Physical Society, 74(9), 855-864.

[24] Shehzad, S.A., Hayat, T., \& Alsaedi, A. (2016). Three-dimensional MHD flow of Casson fluid in porous medium with heat generation. Journal of Applied Fluid Mechanics, 9(1), 215-223.

[25] Eid, M.R., Mahny, K.L., Muhammad, T., \& Sheikholeslami, M. (2018). Numerical treatment for Carreau nanofluid flow over a porous nonlinear stretching surface. Results in Physics, $8,1185-1193$ 